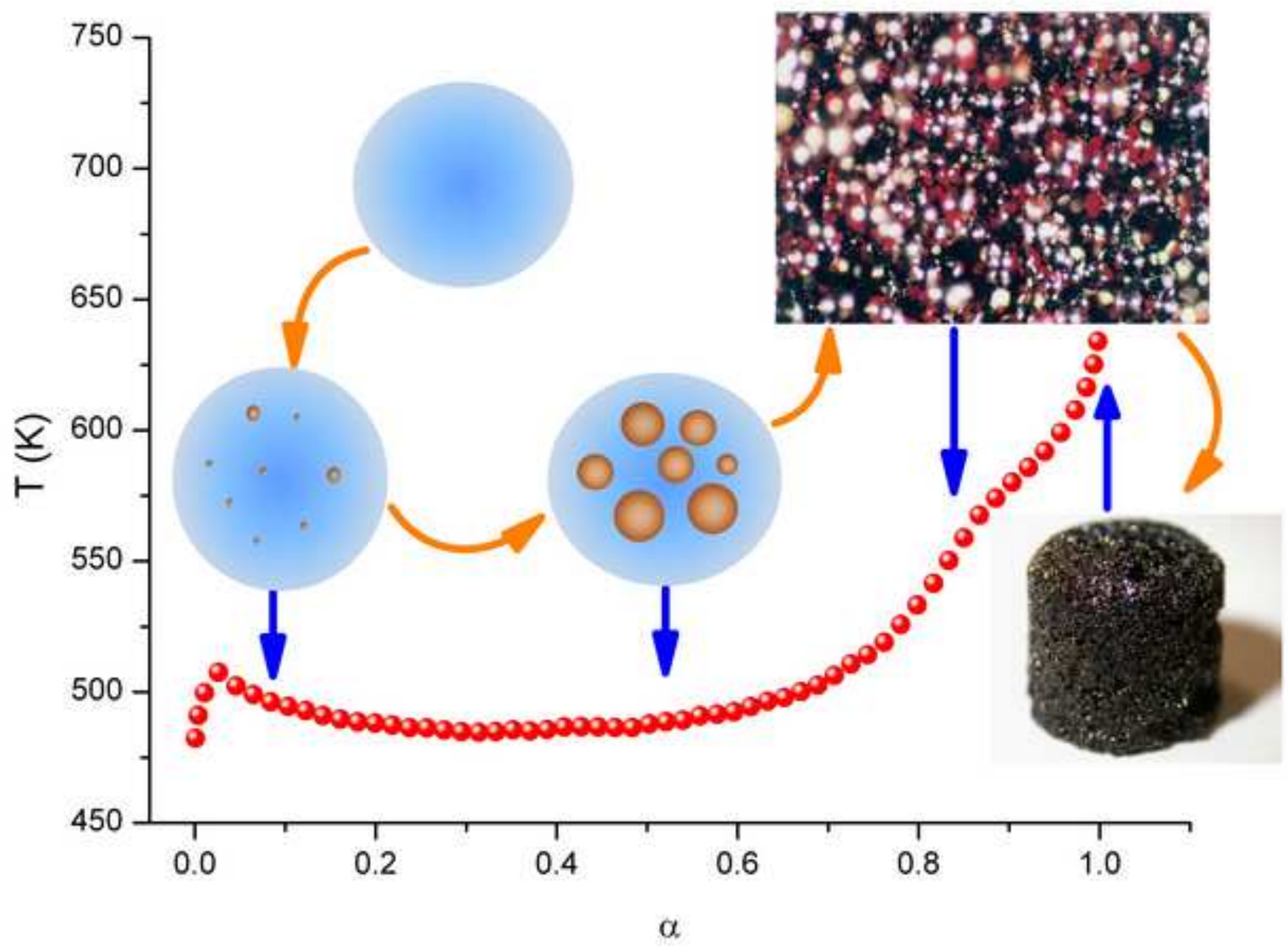




\section{Kinetic Model for Thermal Dehydrochlorination of Poly(vinyl chloride)}

Pedro E. Sánchez-Jiménez, Antonio Perejón, José M. Criado, María J. Diánez, and Luis A. Pérez-Maqueda*

Instituto de Ciencia de Materiales de Sevilla (C.S.I.C. - Universidad de Sevilla)

Americo Vespucio 49, Sevilla, Spain

\section{ABSTRACT}

In this paper, a novel method for calculating degradation kinetics is presented. The method has been applied to the thermal dehydrochlorination of two different samples of PVC. It has been observed that this dehydrochlorination is complex and involves two different processes. A model that accounts for the entire dehydrochlorination is proposed. This model involves nucleation and growth and diffusion controlled mechanisms. The kinetic parameters are obtained from linear heating rate, isothermal and sample controlled thermal analysis experiments. Kinetic results obtained from the macroscopic thermal analysis measurements demonstrate the correlation between the kinetics of the thermal dehydrochlorination of PVC and the structure of this macromolecule.

KEYWORDS: PVC, dehydrochlorination, kinetics, kinetic model, nucleation and growth, diffusion

*corresponding author: maqueda@cica.es 


\section{INTRODUCTION}

Poly(vinyl chloride) (PVC) is one of the most common polymers because its properties and low cost and, therefore, it has been extensively studied in literature. Due to the low thermal stability of this polymer, many studies deal with its thermal degradation. In general, it is established in literature that the thermal decomposition of PVC takes place in two processes [1-6]. The first one corresponds almost exclusively to a side group elimination or dehydrochlorination of the PVC leaving a conjugated polyene structure. Most of the $\mathrm{Cl}$ is released as $\mathrm{HCl}$ that represents up to $99 \%$ of the total volatiles; this $\mathrm{HCl}$ is accompanied with a small amount of hydrocarbons, mainly aromatics such as benzene $[3-5,7-16]$. The relatively low thermal stability of PVC has been attributed by many researchers to the presence of thermally labile structural segments or defects that are responsible of this instability. Among those defects, the most important ones are structural defects such as branching, tertiary chlorine, unsaturations, oxygen containing groups, head-to-head structures and polymerization residues $[14,17-$ 32]. Due to the importance of defects from the point of view of the thermal degradation of PVC, several articles deal with the formation of these defects in PVC macromolecules [18, 31, 33]. Additionally, some authors have recognized the importance of the secondary molecular structure, namely the tacticity governed microstructure, on the thermal degradation of PVC. Thus, it has been claimed that the GTTG $^{-}$ conformer of the mm triads that occur in $m m r$ segments of the polymer are responsible of the thermal instability [34-37].

The kinetics of the thermal dehydrochlorination of PVC has been extensively studied in literature using both isothermal and linear heating rate methods [12, 13, 38-57]. It is striking the very broad dispersion of results in terms of kinetic parameters reported in these papers. Most of the kinetic studies of thermal dehydrochlorination of PVC assume an $n$ order kinetic model for the analysis $[12,13,38-55$, 58-62]. Additionally, some authors have used a more complex approach by proposing either two consecutive $[8,13,50]$ or two competitive [49] $n$ order kinetic models. The use of $n$ order kinetic equations for describing solid state reactions is a very simple assumption commonly used in thermal 
degradation of polymers. Thus, it is assumed that the reaction rate is proportional to the reacted fraction (or unreacted material) to the power of $n$, where $n$ is the reaction order. From the formal point of view, the $n$ order kinetic model has only physical meaning for $n$ values equal to $1,3 / 4$ and $1 / 2$. For these values, the $n$ order kinetic model corresponds to a phase boundary-controlled reaction contracting in one, two and three dimensions, respectively. Intermediate values could correspond to a mixed situation due to, for example, mixtures of particles with different morphologies [63]. In any case, for most of the polymer degradation studies, the $n$ order kinetic models are used just as a fitting function without physical. In fact, the $n$ parameter might have any value that fits the experimental curves, and no conclusions are drawn from the obtained values. It is worth noting that the assumption of a kinetic mechanism produces a significant effect on the kinetic parameters, i.e. activation energy and preexponential factor, resulting from the kinetic analysis. Thus, if an incorrect kinetic model is assumed, the resulting parameters will also be erroneous. Recently, it has been reanalyzed the kinetics of the thermal dehydrochlorination of PVC and it has been concluded that, despite the general believe, this process cannot be described by an $n$ order kinetic model but by a mechanism of nucleation and growth of nuclei.[56] More recently, Simoes et al have reached a similar conclusion in terms of the kinetics of this thermal degradation reaction when studying different PVC samples [57]. Nevertheless, the nucleation and growth of nuclei model does not describe the entire dehydrochlorination process, and deviations in the activation energy and the Arrhenius pre-exponential factor for large values of $\alpha$, i.e. $\alpha>0.7$, are observed. In addition, non-conventional thermal methods such as constant rate thermal analysis [64, 65], stepwise isothermal analysis [65] and high-resolution thermogravimetry [66], that provide a better resolution power than conventional procedures, have shown that the thermal dehydrochlorination of PVC is not a simple process but a complex one that involves two weight steps. These two weight steps have been attributed to different syndiotacticities in the PVC macromolecules, in agreement with results obtained from FTIR [66].

The objective of the present work is the study of the thermal dehydrochlorination of two different samples of PVC and the proposal of a kinetic mechanism that accounts for the entire complex process. 
Kinetic parameters will be obtained from linear heating rate, isothermal and sample controlled thermal analysis experiments.

\section{THEORETICAL}

In a solid-state reaction, the general kinetic equation that describes the reaction rate is usually written as follows:

$$
\frac{d \alpha}{d t}=A \exp (-E / R T) f(\alpha)
$$

where $\alpha$ is the reacted fraction, $t$ is the time, $\mathrm{d} \alpha / \mathrm{d} t$ is the reaction rate, $A$ is the Arrhenius pre-exponential factor, $R$ is the gas constant, $E$ the activation energy, $T$ is the process temperature and $f(\alpha)$ accounts for the reaction rate dependence on $\alpha$. The kinetic model, $f(\alpha)$ is an algebraic expression which is usually associated with a physical model that describes the kinetics of the solid state reaction. Table 1 shows the functions corresponding to the most commonly used mechanisms found in literature.

Recently, it has been reported that a modified Sestak-Berggren equation, i.e. $f(\alpha)=c \alpha^{\mathrm{m}}(1-\alpha)^{\mathrm{n}}$, with values for $\mathrm{n}$ and $\mathrm{m}$ in the range from 0.4 to 0.8 and -1 and 0.7 , respectively, not only fits every kinetic ideal model proposed for solid state reactions, but the deviations of the ideal kinetic models due to, for example, inhomogeneities in particle size and shape [63]. Thus, the use of this equation does not limit the kinetic analysis to the use of only ideal kinetic models that might yield erroneous results. Thus, it has been stated in literature that if the kinetic model is discriminated from a predefined set of kinetics models, one of them will be the "best model" even if the set does not contain the true model[67]. This is especially important for the analysis of real samples. The kinetic models reported in literature have been proposed by assuming ideal conditions in terms of reaction driving force and particle size and shape. Thus, it has been observed that deviations from these ideal situations will definitely produce a change in the form of the $f(\alpha)$ functions, and the experimental curves could not be properly fitted by the ideal models. Nevertheless, the use of the modified Sestak-Berggren equation instead of the equations corresponding to ideal models will fit the data, not only, when they are described by one of the proposed 
ideal kinetic models, but when they follow a different mechanism not corresponding to any of the ideal ones. Thus, equation (1) can be written as

$$
\frac{d \alpha}{d t}=c A \exp (-E / R T) \alpha^{m}(1-\alpha)^{n}
$$

Equations (1) and (2) are general expressions that describe the relationship among the reaction rate, reacted fraction and temperature independently of the thermal pathway used for recording the experimental data. In the case that the experimental data were recorded at a constant heating rate $\beta=d T / d t$, equation (2) would be written as follows:

$$
\frac{d \alpha}{d T}=\frac{c A}{\beta} \exp (-E / R T) \alpha^{m}(1-\alpha)^{n}
$$

For experiments performed under isothermal conditions, the sample temperature is rapidly increased up to a certain temperature and maintained at this temperature while the reaction evolution is recorded as a function of the time. Under these experimental conditions, the term $A \exp (-E / R T)$ remains constant at a value $k$, and therefore equation (2) becomes:

$$
\frac{d \alpha}{d t}=c k(\alpha)^{m}(1-\alpha)^{n}
$$

Sample Controlled Thermal Analysis (SCTA) is another alternative approach which is attracting a rising interest for decomposition reactions.[64, 68-70] In SCTA experiments, the evolution of the reaction rate with the time is predefined by the user and, most usually, it is maintained at a constant value, $\mathrm{C}=\mathrm{d} \alpha / \mathrm{dt}$, along the entire process. In this case, the technique is named Constant Rate Thermal Analysis (CRTA). This way, by selecting a decomposition rate that is slow enough, the mass and heat transfer phenomena occurring during the reaction are minimized, what is an useful asset when dealing 
with reactions as complex as polymer pyrolysis. Thus, the results obtained by CRTA are more representative of the forward reaction than those resulting from more conventional methods $[68,69,71$ 73].

Under constant rate thermal analysis (CRTA) conditions, equation (2) becomes:

$$
C=c A \exp (-E / R T)(\alpha)^{m}(1-\alpha)^{m}
$$

\section{Model-free methods}

Model-free methods are used for determining the activation energy as a function of the reacted fraction without any previous assumption on the kinetic model fitted by the reaction. The Friedman isoconversional method [74] is a widely used differential method that, unlike conventional integral model-free methods, provides accurate values of activation energies even if the activation energy were a function of the reacted fraction [75].

Equation (1) can be written in logarithmic form:

$$
\ln \left(\frac{d \alpha}{d t}\right)=\ln (A f(\alpha))-\frac{E}{R T}
$$

Moreover, at a constant value of $\alpha, f(\alpha)$ would be also constant and equation (6) would be written in the form:

$$
\ln \left(\frac{d \alpha}{d t}\right)_{\alpha}=\text { Const }-\frac{E_{\alpha}}{R T_{\alpha}}
$$

The activation energy at a constant $\alpha$ value, $E_{\alpha}$, can be determined from the slope of the plot of the left hand side of equation (7), that is the logarithm of the reaction rate at a constant value of $\alpha$, against the inverse of the temperature at the same value of $\alpha$. 


\section{EXPERIMENTAL}

Two samples of PVC with different molecular weight purchased from Aldrich have been used in this study:

Sample 1 (Aldrich 389323): average $\mathrm{Mn} \sim 47,000$; average $\mathrm{Mw} \sim 80,000$; fine powder.

Sample 2 (Aldrich 346764): average $\mathrm{Mn} \sim 99,000$; average $\mathrm{Mw} \sim 233,000$; fine powder.

The samples were used as received without any further modification.

A homemade thermogravimetric instrument that allows working, not only under conventional linear heating and isothermal conditions, but also under sample controlled thermal analysis conditions has been used for the present study. A detailed description of the instrument can be found in references [64, 65]. In order to avoid heat and mass transfer phenomena, very small amounts of sample were used. Thus, about $10 \mathrm{mg}$ and $25 \mathrm{mg}$ of sample were used for the experiments under conventional conditions (linear heating rate and isothermal) and sample controlled thermal analysis conditions, respectively. Additionally, the reproducibility of the measurements was checked by repeating experiments with different amounts of material. In the case of the sample controlled conditions, a slightly larger amount of sample was used to obtain a better control of the reaction rate. Nevertheless, it has to be taken into consideration that under these experimental conditions, the heat and mass transfer phenomena are minimized because the reaction rate is controlled all over the process. All experiments were performed in nitrogen atmosphere (flow: $100 \mathrm{cc} \min ^{-1}$ ).

The evolved gas analysis was performed under high vacuum using another homemade instrument with a reactor and a quadrupole mass spectrometer (Prism, Pfeiffer) [76]. In this case, $3.5 \mathrm{mg}$ of sample was used for the experiment. 


\section{RESULTS}

Fig. 1 shows the thermogravimetric curves, in its integral and differential forms, corresponding to the thermal dehydrochlorination of PVC sample 1 under different linear heating rates. Weight losses have been normalized, as it is commonly done for kinetic analysis of solid state reaction, and the process is described in terms of reaction fraction or $\alpha$. Nevertheless, the weight loss for all curves in Fig. 1 was about $59 \%$ that is the stoichiometric value corresponding to the release of $\mathrm{HCl}$ due to total thermal dehydrochlorination of PVC, in agreement with previous literature data [3-5, 7-16]. It is quite clear from Fig. 1 that curves show a shoulder for all heating rates. This behaviour suggests a complex dehydrochlorination mechanism. In addition the evolution of the $\mathrm{HCl}$ mass signal recorded by mass spectrometry as a function of the temperature shows that $\mathrm{HCl}$ is evolved in two overlapping stages (Fig. 2), corresponding to both weight losses in the TG traces (Fig. 1). It is noteworthy to point out that, although thermogravimetric results show that both processes are not totally resolved (Fig. 1), the shoulder is observed at a reacted fraction, $\alpha$, roughly closed to 0.6 independently of the heating rate selected, what suggests that thermal dehydrochlorination of PVC takes place through two independent reactions. This behaviour is not consistent with the proposal of $\mathrm{Wu}$ et al [49] that consider that the thermal dehydrochlorination of PVC is described by two competitive processes. In the case that a solidstate reaction is described by two competitive processes with different kinetic parameters, the contribution of each of them will depend on temperature and due to the fact that a change in the heating rate produces a shift in the average temperature at which the process takes place, the contribution of each individual process to the overall reaction will be different for the different heating rates [75]. Moreover, the activation energy values plotted in Fig. 3 as a function of the reaction progress as calculated by means of the model free method previously described in the theoretical section seems to support this conclusion. Thus, it is clear from Fig. 3 that the activation energy remains near constant at a value close to $125 \mathrm{~kJ} / \mathrm{mol}$ until the reacted fraction at the shoulder is reached, while for higher values of $\alpha$ the activation energy sharply increases up to values larger than $190 \mathrm{~kJ} \mathrm{~mol}^{-1}$. It would be expected that 
these two limit values of the activation energy would be close to the activation energies corresponding to every one of the two independent overlapping reactions.

Taking into account the above considerations, the total reaction rate would be given by the addition of the two reaction rates corresponding to each independent process multiplied by the fraction representing the contribution of each of them to the overall decomposition reaction. From the kinetic point of view, the total reaction rate would be formulated, according to equation (3), by the following equation:

$$
\frac{d \alpha}{d T}=\frac{1}{\beta}\left[l_{1} c_{1} A_{1} \exp \left(-E_{1} / R T\right) \alpha_{1}^{m 1}\left(1-\alpha_{1}\right)^{n 1}+l_{2} c_{2} A_{2} \exp \left(-E_{2} / R T\right) \alpha_{2}^{m 2}\left(1-\alpha_{2}\right)^{n 2}\right]
$$

where the subscripts 1 and 2 refer to the first and second process, respectively, $l_{1}$ and $l_{2}$ being the contribution fraction of the first and second process to the overall reaction. It is evident that $l_{1}$ and $l_{2}$ must accomplish the following relationships:

$$
\begin{gathered}
l_{1}+l_{2}=1 \\
l_{1} \alpha_{1}+l_{2} \alpha_{2}=\alpha
\end{gathered}
$$

All the parameters in equation (8) are identified by comparing the experimental curves with those resulting from solving equation (8) and (9) using iterative optimization. Thus, a nonlinear least-squares algorithm is used to determine the variable parameters, i.e. $l_{1},\left(c_{1} A_{1}\right), E_{1}, m_{1}, n_{1}, l_{2},\left(\mathrm{c}_{2} \mathrm{~A} 2\right) E_{2}, m_{2}$ and $n_{2}$, which minimize the values of the objective function $F$ given below (equation (10)).

$$
F=\sum_{j=1}^{N} \sum_{i=1}^{M_{j}} \frac{1}{M_{j}}\left(\left(\frac{d x}{d T}\right)_{j, i}^{\text {experimental }}-\left(\frac{d \propto x}{d T}\right)_{j, i}^{\text {calculated }}\right)^{2}
$$

where $N$ is the total number of experimental curves, $j$ is the curve index, $i$ is the experimental point index, $M_{j}$ is the total number of experimental points in the experimental curve $j,\left(\frac{d x}{d T}\right)_{j, i}^{\text {experimental }}$ is the experimental value corresponding to the curve $j$ in the point $i$, and $\left(\frac{d x}{d T}\right)_{j, i}^{\text {calculated }}$ is the calculated value for the curve $j$ in the point $i$. 
The experimental curves included in Fig. 1 have been simultaneously fitted from the above analysis procedure leading to the following kinetic parameters: $l_{1}=0.657\left(\right.$ i.e.; $65.7 \%$ ), $E_{1}=113.1 \mathrm{~kJ} \mathrm{~mol}^{-1}$ , $\left(c_{1} A_{1}\right)=610^{10} \mathrm{~min}^{-1}, n_{1}=1.16, m_{1}=0.683, l_{2}=0.343$ (i.e.; $\left.34.3 \%\right), E_{2}=202.1 \mathrm{~kJ} \mathrm{~mol}^{-1},\left(c_{2} A_{2}\right)=10^{17}$ $\min ^{-1}, n_{2}=1.36$, and $m_{2}=-1.88$. It must be pointed out that the two values of activation energies obtained roughly agree with those calculated from the Friedman for low and high values of alpha, respectively (Fig. 3) as previously forecasted. Fig. 4 shows the comparison of the modified SestakBerggren $f(\alpha)$ functions resulting from the kinetic analysis with some of the conversion functions often used in the literature, which are listed in Table 1. It is clear from this figure that the conversion function associated with the thermal degradation of PVC closely follows for the first process a nucleation kinetic model, in particular a A2 model. Besides, the second process follows a diffusion kinetic model, although it cannot be properly described by any of the ideal ones.

The simulation of curves obtained with the calculated kinetic parameters is a useful method for checking the results obtained by this kinetic analysis. Thus, a set of curves have been simulated assuming identical heating conditions as those used in the experiments and the kinetic parameters obtained from the kinetic analysis. The simulations have been performed by numerical integration of equation (8). As it is shown in Fig. 1, both the reconstructed (simulated) and the experimental curves overlap, validating the kinetic parameters obtained from the analysis. To further check the kinetic parameters, other linear heating rate experiments at 20 and $40 \mathrm{~K} \mathrm{~min}^{-1}$ have been recorded (Fig. 5). These curves are perfectly matched by the simulated curves constructed by assuming these linear heating rate conditions and the kinetic parameters obtained from the kinetic analysis. An additional test was performed by recorded an isothermal experiment at $543 \mathrm{~K}$ (Fig. 6). As in the previous cases, a curve have been simulated with the calculated kinetic parameters and for a constant temperature of 543 $K$ and the resulting curve also perfectly matches the experimental data (Fig. 6). Finally, it has been considered of interest to perform an experiment under Sample Controlled Thermal Analysis (SCTA) conditions. Thus, Fig. 7 includes the experimental curve resulting from the thermal dehydrochlorination of PVC at a constant reaction rate of $610^{-3} \mathrm{~min}^{-1}$. It has been stated in literature that the SCTA method 
provides a much better resolution power for the kinetic model of solid state reactions than conventional linear heating or isothermal conditions.[77, 78] Thus, the shape of the curves is very much dependent on the kinetic model followed by the process, unlike curves under linear heating rate conditions that always have a sigmoid shape. The SCTA experiment corresponding to the dehydrochlorination of the PVC shows for the initial part a temperature decrease until a certain conversion value is reached, followed by a rising until completing the reaction. Thus, the curve falls back on itself upon achieving the preset constant rate. This shape is very characteristic of a nucleation and growth mechanism [77-79]. For the second process at higher temperature, it is hard to discriminate the shape of the curve because there is still some overlapping, although smaller than in conventional linear heating experiments, with the other process. In any case, it seems to have a sigmoid shape that is characteristic of diffusion controlled mechanisms $[77,78]$. Thus, a curve was simulated using the kinetic parameters obtained from the above analysis and assuming SCTA conditions identical to those of the experimental curve in Fig. 7. The resulting simulated curve matches very nicely the experimental one, validating the kinetic parameters. The fact that the kinetic parameters obtained from a set of linear heating rate curves are able to reconstruct curves obtained under quite different heating conditions, i.e. other linear heating rates, isothermal and sample controlled, not only validates the kinetic parameters, but also suggests that mass and heat transfer phenomena have been successfully minimized, because otherwise the shapes of the curves would be influenced by the experimental arrangement.

A similar study has been performed on PVC sample 2. Fig. 8 includes the TG-DTG traces obtained for this sample under different linear heating rates. Curves look quite similar to those corresponding to the sample 1 despite their different weight numbers. As for sample 1, identical kinetic analysis procedure was performed. The activation energy values as a function of the reaction progress obtained by means of the model free method described in the theoretical section are included in Fig. 9. As for sample 1, the activation energy does not remain constant for all the values of the reacted fraction indicating that it is a complex process, and the activation energy that remains almost unchanged at about $122 \mathrm{~kJ} \mathrm{~mol}^{-1}$ for values of the reacted fraction smaller than 0.6 increases sharply up to more than $190 \mathrm{~kJ}$ 
$\mathrm{mol}^{-1}$ for larger values of the reacted fraction (Fig. 9). The kinetic analysis described in the experimental section yields the following kinetic parameters: $l_{1}=0.629, E_{1}=114.5 \mathrm{~kJ} \mathrm{~mol}^{-1},\left(\mathrm{c}_{1} \mathrm{~A}_{1}\right)=610^{10} \mathrm{~min}^{-1}$, $n_{1}=1.19, m_{1}=0.695, l_{2}=0.371, E_{2}=202.0 \mathrm{~kJ} \mathrm{~mol}^{-1},\left(\mathrm{c}_{2} \mathrm{~A}_{2}\right)=10^{17} \mathrm{~min}^{-1}, n_{2}=1.27$, and $\mathrm{m}_{2}=-1.41$. These kinetic parameters are quite similar to those obtained for sample 1. In addition, Fig. 10 shows the comparison of the modified Sestak-Berggren $f(\alpha)$ functions resulting from the analysis with some of the conversion functions in Table 1. As also observed for sample 1, it is clear from this figure that the conversion function associated with the thermal degradation of PVC closely follows a A2 nucleation kinetic model for the first process and a diffusion controlled model for the second one. In this latter case, although the shape is characteristic of the diffusion models, it does not match any of the ideal ones.

The set of curves simulated by assuming the kinetic parameters resulting from the analysis closely match the experimental curves in Fig. 8, validating the analysis. In addition, it was considered of interest, as for sample 1, to obtain a set of curves under different experimental conditions than those used for the kinetic analysis. Thus, four curves, two under linear heating rate conditions, i.e. $\beta=20$ and $40 \mathrm{~K} \mathrm{~min}^{-1}$ (Fig. 11), another under isothermal conditions, i.e. $\mathrm{T}=543 \mathrm{~K}$ (Fig. 12), and the last one under sample controlled conditions, i.e. $\mathrm{C}=610^{-3} \mathrm{~min}^{-1}$ (Fig. 13) were obtained experimentally. The fact that the curves simulated using the kinetic parameters obtained from the previous analysis match those obtained experimentally (Fig. 11-13) confirms the validity of the kinetic parameters.

These results contrast with those previously reported in literature, that describe the thermal dehydrochlorination of PVC by means of $\mathrm{n}$ order kinetic model. Nevertheless, it has to be taken into consideration that in those previous studies an $\mathrm{n}$ order kinetic model had been assumed. In fact, these $\mathrm{n}$ order kinetic models are generally used for describing polymer degradation reactions. Nevertheless, this assumption is not necessarily true, and it has been shown in previous works that, while some polymer degradation reactions could be described by a n order model, other, such as that of polyethylene, follow quite different kinetic models.[80] Several authors have already warned about the risk of assuming predefined kinetic models for solid state reactions [67, 81, 82]. Thus, even when the kinetic model is 
selected from a limited number of models, necessarily, one of them will provide the best fit even if it is not the true one [67]. The very broad dispersion of data reported in literature for the thermal dehydrochlorination of PVC can be understood by considering that the resulting activation energy and preexponential factor are very much dependent of the kinetic model assumed.

In the present study, it has been clearly shown that the PVC dehydrochlorination is a complex process involving two different chlorine groups. Both groups might be related to sequences with different tacticities in agreement with previous papers that claim a significant contribution of the stereochemical composition on the degradation rate. Thus, it has been reported that an increase of polymer syndiotacticity provokes a decrease of labile chlorine groups [32, 34-37]. Recently, it has been studied the tacticity of quite different pure PVC samples by FTIR and it has been concluded that the ratio between syndiotactic short sequences and syndiotactic long sequences are in the range from 1.86 to 1.44, while these ratios are coincident with the two weight losses observed for the dehydrochlorination by high resolution thermogravimetry [66]. Those ratios are consistent with the percentages obtained in the present paper for the two processes, i.e. $65.7 \%$ and $62.9 \%$ for the first one and $34.3 \%$ and $37.1 \%$ for the second one, suggesting a different stereochemistry for two different chlorine groups that contribute to each of them. The kinetic study has shown that both dehydrochlorination processes follow a different kinetic model. Thus, the first one involves a nucleation and growth model whereas the second one involves a diffusion controlled model. Both processes overlap from the first stages of degradation (Fig. 1 and 8), but the contribution of the nucleation and growth one is much larger in the lower temperature range, while the higher temperature range is almost totally described by the diffusion one. It is quite interesting that one of these two processes takes place through a nucleation and growth model. As a matter of fact, these kind of kinetic models are commonly found in crystallization processes but are rarer for other solid state reactions. Nevertheless, processes such as the reduction of $\mathrm{V}_{2} \mathrm{O}_{5}, \mathrm{Fe}_{2} \mathrm{O}_{3}$, and $\mathrm{Fe}_{3} \mathrm{O}_{4}[79,83]$ or the thermal decomposition of nickel nitrate [84] follow this kind of kinetic model. The thermal dehydrochlorination of PVC is the only example, to our knowledge, in which this kind of kinetic model has been reported in the field of thermal degradation of polymers. From the physical 
point of view, the nucleation and growth model implies that the reaction starts in discrete points, called nuclei, and the progress of the reaction follows from these nuclei into the rest of the material. This result agrees with the assumption that PVC decomposes starting from irregularities or defects where the chlorine groups are especially labile. Thus, these defects can act as the nuclei where decomposition starts, while the decomposition continues in two dimensions along the rest of the sample. This kinetic model is in agreement with the fact that lower syndiotacticity involves larger concentration of structural defects $[32,66]$. From the practical point of view, it is quite significant that the thermal dehydrochlorination follow such kinetic model. Thus, it means that if the material goes through a high temperature event during processing, recycling or any application, the nucleation and growth process will be already initiated and the degradation will be speeded up. This behaviour can be illustrated with the SCTA experiments in Fig. 7 and 13, where the reaction rate has been automatically maintained constant along the entire process. Thus, at the beginning of the dehydrochlorination, temperature has to be increased up to a relatively high value to initiate the process, while once this first stage is surpassed, the temperature has to decrease for maintaining the reaction rate constant. Furthermore, the second dehydrochlorination process was described by a diffusion controlled process. This diffusion controlled process was also confirmed by observations with the optical microscope. Thus, Fig. 14a includes the micrograph of the PVC sample 1 dehydrochlorinated up to an extend of $70 \%$, that corresponds to the first part of the second process, while Fig. 14b includes the micrograph corresponding to the same sample totally dehydrochlorinated. The sample partially dehydrochlorinated shows the presence of a large number of bubbles all over the sample, while the sample totally dehydrochlorinated has a globular or foam type structure with an apparent density of $0.1 \mathrm{~g} \mathrm{~cm}^{-3}$. Thus, during the last part of the dehydrochlorination, it seems that $\mathrm{HCl}$ gasses are occluded within the material due to the formation of a carcass that hinders the gas release. Therefore, in this stage, the diffusion of the gas from the bulk to the surface is the limiting parameter of kinetics. Finally, after the total dehydrochlorination, the foam or globular structure is preserved. This finding agrees with the results of Dadvand et al[10] that, after studying the thermal dehydrochlorination of PVC by gas chromatography and mass spectrometry, 
suggest that certain degree of cross-linking is developed impeding the escape of the $\mathrm{HCl}$ in the final stage of dehydrochlorination. As a matter of fact, in PVC the cross-linking, whereby there is a creation of bonds between two adjacent polymer chains, generates a more compact, less easily volatilized structure [85]. Interestingly, a similar behaviour has been reported for the pyrolysis of rice husk, where a high thermal stability $\mathrm{Si}-\mathrm{O}$ carcass is formed and gives rise to a diffusion controlled process, in such a way that the resulting material presents a typical globular structure [86]. From the practical point of view, the presence of this diffusion controlled process determines the globular like or foam microstructure of the residue. As a matter of fact, some authors have proposed the pyrolysis of PVC as a procedure to prepare porous carbon materials without further activation [87].

In conclusion, the thermal dehydrochlorination of PVC is complex and involves two different processes: a first process with an activation energy of about $114 \mathrm{~kJ} \mathrm{~mol}^{-1}$ that follows a nucleation and growth type kinetic model and a second process with an activation energy of about $202 \mathrm{~kJ} \mathrm{~mol}^{-1}$ that follows a diffusion controlled kinetic model. The first process that follows a nucleation and growth mechanism could be related with the presence of the well described labile sites in PVC where the reaction starts and follows from these nuclei into the rest of the material. The second process follows a diffusion controlled model that was supported by microscopic observation of the material that shows the formation of bubbles due to the release of the $\mathrm{HCl}$ during the last steps of dehydrochlorination. The kinetics of two samples with different molecular weight resulted quite similar.

\section{ACKNOWLEDGMENT}

Financial support from projects TEP-03002 from Junta de Andalucía and MAT 2008-06619/MAT from the Spanish Ministerio de Ciencia e Innovación is acknowledged.

\section{REFERENCES}

1. Omara MM. Journal of Polymer Science Part a-1-Polymer Chemistry 1970;8(7):1887-\&.

2. Guyot A and Bert M. Journal of Applied Polymer Science 1973;17(3):753-768.

3. Omara MM. Pure and Applied Chemistry 1977;49(5):649-660. 
4. Montaudo G and Puglisi C. Polymer Degradation and Stability 1991;33(2):229-262.

5. Petre AL, Budrugeac P, and Segal E. Journal of Thermal Analysis and Calorimetry 1999;56(3):1065-1070.

6. Yanborisov VM, Minsker KS, Zaikov GE, and Zaikov VG. Journal of Vinyl \& Additive Technology 2002;8(3):176-179.

7. Miranda R, Pakdel H, Roy C, and Vasile C. Polymer Degradation and Stability 2001;73(1):4767.

8. Miranda R, Yang J, Roy C, and Vasile C. Polymer Degradation and Stability 1999;64(1):127144.

9. Pielichowski K, Hamerton I, Pielichowski J, and Stanczyk P. European Polymer Journal 1998;34(5-6):653-657.

10. Dadvand N, Lehrle RS, Parsons IW, and Rollinson M. Polymer Degradation and Stability 1999;66(2):247-255.

11. Pielichowski K and Njuguna J. Thermal degradation of polymeric materials. Shawbury, UK: Rapra Technology, 2005.

12. Slapak MJP, van Kasteren JMN, and Drinkenburg AAH. Computational and Theoretical Polymer Science 2000;10(6):481-489.

13. Soudais Y, Moga L, Blazek J, and Lemort F. Journal of Analytical and Applied Pyrolysis 2007;78(1):46-57.

14. Troitskii BB and Troitskaya LS. European Polymer Journal 1999;35(12):2215-2224.

15. Zhu HM, Jiang XG, Yan JH, Chi Y, and Cen KF. Journal of Analytical and Applied Pyrolysis 2008;82(1):1-9.

16. Benes M, Placek V, Matuschek G, Kettrup A, Gyoryova K, and Balek V. Journal of Applied Polymer Science 2006;99(3):788-795.

17. Starnes WH. Progress in Polymer Science 2002;27(10):2133-2170.

18. Wieme J, Reyniers MF, and Marin GB. Macromolecules 2009;42(20):7797-7810.

19. Wypych G. PVC Degradation and Stabilization. Ontario, Canada: ChemTec Publishing, 2008.

20. Minsker KS, Zaikov GE, and Zaikov G. Macromolecular Symposia 2005;228:299-313.

21. Troitskii BB and Troitskaya LS. European Polymer Journal 1997;33(8):1289-1294.

22. Troitskii BB, Yakhnov AS, Novikova MA, Ganyukhina TG, and Denisova VN. European Polymer Journal 1997;33(4):505-511.

23. Starnes WH. Journal of Polymer Science Part a-Polymer Chemistry 2005;43(12):2451-2467.

24. Hjertberg T and Sorvik EM. Polymer 1983;24(6):673-684.

25. Braun D. Progress in Polymer Science 2002;27(10):2171-2195.

26. Ivan B. Thermal stability, degradation, and stabilization mechanisms of poly(vinyl chloride). In: Clough RL, Billingham NC, and Gillen KT, editors. Polymer Durability - Degradation, Stabilization, and Lifetime Prediction, vol. 249, 1996. pp. 19-32.

27. Minsker KS. International Journal of Polymeric Materials 1994;24(1-4):235-251.

28. Yassin AA and Sabaa MW. Journal of Macromolecular Science-Reviews in Macromolecular Chemistry and Physics 1990;C30(3-4):491-558.

29. Zaikov GE, Gumargalieva KZ, Pokholok TV, and Moiseev YV. Oxidation Communications 1997;20(3):323-388.

30. Saeki Y and Emura T. Progress in Polymer Science 2002;27(10):2055-2131.

31. Purmova J, Pauwels KFD, van Zoelen W, Vorenkamp EJ, Schouten AJ, and Coote ML. Macromolecules 2005;38(15):6352-6366.

32. Rogestedt M and Hjertberg T. Macromolecules 1993;26(1):60-64.

33. Van Cauter K, Van den Bossche BJ, Van Speybroeck V, and Waroquier M. Macromolecules 2007;40(4):1321-1331.

34. Millan J, Martinez G, Jimeno ML, Tiemblo P, Mijangos C, and Gomezelvira JM. Makromolekulare Chemie-Macromolecular Symposia 1991;48-9:403-417.

35. Martinez G, Gomezelvira JM, and Millan J. Polymer Degradation and Stability 1993;40(1):1-8. 
36. Millan JL, Martinez G, GomezElvira JM, Guarrotxena N, and Tiemblo P. Polymer 1996;37(2):219-230.

37. Guarrotxena N. On the secondary molecular structures dependences of physical behaviour of polymers. 2. Application to thermal relaxation, aging and basic electrical poperties of poly(vinylchloride( (PVC) and polypropylene In: Zaikov G, Lobo V, and Guarrotxena N, editors. International Perspectives on Chemistry and Biochemical Research. Hauppauge, NY, USA: Nova Science Publisher, 2003. pp. 15.

38. Stromberg RR, Straus S, and Achhammer BG. J. Polym. Sci. 1959;35(129):355.

39. Abbas KB and Sorvik EM. Journal of Applied Polymer Science 1973;17:3567-3576.

40. Danforth JD and Takeuchi T. J Polym Sci Part A-1 Polym Chem 1973;11(9):2091-2098.

41. Basan S and GÃ/4ven O. Thermochimica Acta 1986;106(C):169-178.

42. Hirschler MM. European Polymer Journal 1986;22(2):153-160.

43. Behnisch J and Zimmermann H. Journal of Thermal Analysis 1988;33(1):191-196.

44. Ahmad Z and Manzoor W. Journal of Thermal Analysis 1992;38(10):2349-2357.

45. Day M, Cooney JD, and Klein C. Journal of Thermal Analysis 1993;40(2):669-676.

46. Jimenez A, Berenguer V, Lopez J, and Sanchez A. Journal of Applied Polymer Science 1993;50(9):1565-1573.

47. Day M, Cooney JD, and Fox JL. Journal of Thermal Analysis 1994;42(2-3):397-424.

48. Hussain R and Mahmood F. J. Chem. Soc. Pak. 1994;16:225-228.

49. Wu C-H, Chang C-Y, Hor J-L, Shih S-M, Chen L-W, and Chang F-W. Canadian Journal of Chemical Engineering 1994;72(4):644-650.

50. Marcilla A and Beltran M. Polymer Degradation and Stability 1995;48(2):219-229.

51. Anthony GM. Polymer Degradation and Stability 1999;64(3):353-357.

52. Jimenez A, Iannoni A, Torre L, and Kenny JM. Journal of Thermal Analysis and Calorimetry 2000;61(2):483-491.

53. Yang J, Miranda R, and Roy C. Using the DTG curve fitting method to determine the apparent kinetic parameters of thermal decomposition of polymers. 1st International Conference on Polymer Modification, Degradation and Stabilisation. Palermo, Italy, 2000. pp. 455-461.

54. Jordan KJ, Suib SL, and Koberstein JT. Journal of Physical Chemistry B 2001;105(16):31743181.

55. Ma SB, Lu J, and Gao JH. Energy \& Fuels 2002;16(2):338-342.

56. Budrugeac P, Segal E, Perez-Maqueda LA, and Criado JM. Polymer Degradation and Stability 2004;84(2):311-320.

57. Simoes PN, Coelho JFJ, Goncalves P, and Gil MH. European Polymer Journal 2009;45(7):19491959.

58. Hjertberg T and Sorvik EM. Journal of Applied Polymer Science 1978;22(9):2415-2426.

59. Wu CH, Chang CY, Hor JL, Shih SM, Chen LW, and Chang FW. Waste Management 1993;13(3):221-235.

60. Knümann R and Bockhorn H. Combust. Sci. Technol. 1994;101(1-6):285-299.

61. Bisi M, Nicolella C, Palazzi E, Rovatti M, and Ferraiolo G. Chemical Engineering and Technology 1994;17(1):67-72.

62. Kim S. Waste Management 2001;21(7):609-616.

63. Perez-Maqueda LA, Criado JM, and Sanchez-Jimenez PE. Journal of Physical Chemistry A 2006;110(45):12456-12462.

64. Criado JM, Perez-Maqueda LA, Dianez MJ, and Sanchez-Jimenez PE. Journal of Thermal Analysis and Calorimetry 2007;87(1):297-300.

65. Dianez MJ, Perez-Maqueda LA, and Criado JM. Review of Scientific Instruments 2004;75(8):2620-2624.

66. Gonzalez N, Mugica A, and Fernandez-Berridi MJ. Polymer Degradation and Stability 2006;91(4):629-633.

67. Brown ME. Journal of Thermal Analysis 1997;49(1):17-32. 
68. Rouquerol J. Journal of Thermal Analysis and Calorimetry 2003;72(3):1081-1086.

69. Perez-Maqueda LA, Criado JM, Subrt J, and Real C. Catal. Lett. 1999;60(3):151-156.

70. Perez-Maqueda LA, Ortega A, and Criado JM. Thermochim. Acta 1996;277:165-173.

71. Sorensen OT and Rouquerol.J. Sample Controlled Thermal Analysis. Budapest: Kluwer, 2003.

72. Koga N and Criado JM. International Journal of Chemical Kinetics 1998;30(10):737-744.

73. Criado JM, Gotor FJ, Ortega A, and Real C. Thermochimica Acta 1992;199:235-238.

74. Friedman HL. Journal of Polymer Science Part C-Polymer Symposium 1964(6PC):183-\&.

75. Criado JM, Sanchez-Jimenez PE, and Perez-Maqueda LA. Journal of Thermal Analysis and Calorimetry 2008;92(1):199-203.

76. Perez-Maqueda LA, Criado JM, and Gotor FI. International Journal of Chemical Kinetics 2002;34(3):184-192.

77. Criado JM and Perez-Maqueda LA. Journal of Thermal Analysis and Calorimetry 2005;80(1):27-33.

78. Perez-Maqueda LA, Ortega A, and Criado JM. Thermochimica Acta 1996;277:165-173.

79. Tiernan MJ, Barnes PA, and Parkes GMB. Journal of Physical Chemistry B 2001;105(1):220228.

80. Sanchez-Jimenez PE, Perez-Maqueda LA, Perejon A, and Criado JM. Polymer Degradation and Stability 2009;94(11):2079-2085.

81. Vyazovkin SV and Lesnikovich AI. Journal of Thermal Analysis 1989;35(7):2169-2188.

82. Reading M, Dollimore D, and Whitehead R. Journal of Thermal Analysis 1991;37(9):21652188.

83. Tiernan MJ, Barnes PA, and Parkes GMB. Journal of Physical Chemistry B 1999;103(2):338345.

84. Gotor FJ, Perez-Maqueda LA, Ortega A, and Criado JM. J. Therm. Anal. Calorim. 1998;53(2):389-396.

85. Hirschler MM. Flammability and Fire Performance. In: Wilkes CE, Summers JW, and Daniels CA, editors. PVC Handbook. München, Germany: Carl Hanser Verlag, 2005. pp. 422.

86. Vlaev LT, Markovska IG, and Lyubchev LA. Thermochimica Acta 2003;406(1-2):1-7.

87. Qiao WM, Song Y, Yoon SH, Korai Y, Mochida I, Yoshiga S, Fukuda H, and Yamazaki A. Waste Management 2006;26(6):592-598. 
TABLE 1. Algebraic expressions for the $f(\alpha)$ functions for the most common mechanisms in solid-state reactions and their corresponding equivalent modified Sestak-Berggren equations.

\begin{tabular}{|c|c|c|c|}
\hline Mechanism & Symbol & $f(\alpha)$ & $\begin{array}{c}\text { Equivalent reduced Sestak- } \\
\text { Berggren equation }\end{array}$ \\
\hline
\end{tabular}

Phase boundary controlled reaction

(contracting area, i. e. bidimensional shape)

R2

$(1-\alpha)^{1 / 2}$

$(1-\alpha)^{1 / 2}$

Phase boundary controlled reaction

(contracting volume, i. e. tridimensional shape)

R3

$$
(1-\alpha)^{2 / 3}
$$

$(1-\alpha)^{2 / 3}$

Unimolecular decay law (instantaneous nucleation and unidimensional growth)

F1

$$
(1-\alpha)
$$

$(1-\alpha)$

Random instant nucleation and twodimensional growth of nuclei
A2

$$
2(1-\alpha)[-\ln (1-\alpha)]^{1 / 2}
$$
$2.079(1-\alpha)^{0.806} \alpha^{0.515}$

(Avrami- Erofeev equation)

Random instant nucleation and threedimensional growth of nuclei

$$
\text { A3 }
$$

$$
3(1-\alpha)[-\ln (1-\alpha)]^{2 / 3} \quad 3.192(1-\alpha)^{0.748} \alpha^{0.693}
$$

(Avrami- Erofeev equation)

Two-dimensional diffusion

(bidimensional particle shape)

D2

$$
1 /-\ln (1-\alpha)
$$

$0.973(1-\alpha)^{0.425} \alpha^{-1.008}$

Three-dimensional diffusion

(tridimensional particle shape)

$$
\text { D3 } \quad \frac{3(1-\alpha)^{2 / 3}}{2\left[1-(1-\alpha)^{1 / 3}\right]}
$$$$
4.431(1-\alpha)^{0.951} \alpha^{-1.004}
$$

Jander equation 


\section{FIGURE CAPTIONS}

Figure 1. Thermogravimetric curves in its integral (top) and differential (bottom) forms corresponding to the thermal dehydrochlorination of PVC sample 1 obtained under different linear heating ratesThe dots are the experimental curves while solid lines are the theoretical ones simulated using the kinetic parameters obtained from the analysis. The contribution of the two processes to the overall reactions are represented by the dashed lines.

Figure 2. Ion current corresponding to $\mathrm{HCl}$ mass signals recorded by mass spectrometry as a function of the temperature for sample 1 at a heating rate of $10 \mathrm{~K} \mathrm{~min}^{-1}$

Figure 3. Activation energy values as a function of the reaction progress obtained by means of the model free method for the PVC sample 1.

Figure 4. Comparison of the modified Sestak-Berggren $f(\alpha)$ functions resulting from the kinetic analysis (points) with some of the conversion functions often used in the literature and listed in Table 1 (lines).

a) first process and b) second process.

Figure 5. Thermogravimetric curves obtained under linear heating rate conditions at (a) 20 and (b) $40 \mathrm{~K}$ $\min ^{-1}$ for the PVC sample 1. Experimental curves are represented by points while the theoretical reconstructed ones are represented by lines.

Figure 6. Isothermal experiment at $543 \mathrm{~K}$ for the PVC sample 1. Experimental curve is represented by points while the theoretical reconstructed one is represented by a line. The inset figure shows a detail of the initial part of the degradation curve.

Figure 7. Sample controlled thermal analysis curve resulting from the thermal dehydrochlorination of PVC sample 1 at a constant reaction rate of $610^{-3} \mathrm{~min}^{-1}$. Experimental curve is represented by points while the theoretical reconstructed one is represented by a line. 
Figure 8. Thermogravimetric curves in its integral (top) and differential (bottom) forms corresponding to the thermal dehydrochlorination of PVC sample 2 obtained under different linear heating rates. The dots are the experimental curves while solid lines are the theoretical ones simulated using the kinetic parameters obtained from the analysis. The contribution of the two processes to the overall reactions are represented by the dashed lines.

Figure 9. Activation energy values as a function of the reaction progress obtained by means of the model free method for the PVC sample 2.

Figure 10. Comparison of the modified Sestak-Berggren $f(\alpha)$ functions resulting from the kinetic analysis (points) with some of the conversion functions often used in the literature and listed in Table 1 (lines). a) first process and b) second process.

Figure 11. Thermogravimetric curves obtained under linear heating rate conditions at (a) 20 and (b) 40 $\mathrm{K} \min ^{-1}$ for the PVC sample 2. Experimental curves are represented by points while the theoretical reconstructed ones are represented by lines.

Figure 12. Isothermal experiment at $543 \mathrm{~K}$ for the PVC sample 2. Experimental curve is represented by points while the theoretical reconstructed one is represented by a line.

Figure 13. Sample controlled thermal analysis curve resulting from the thermal dehydrochlorination of PVC sample 2 at a constant reaction rate of $610^{-3} \mathrm{~min}^{-1}$. Experimental curve is represented by points while the theoretical reconstructed one is represented by a line.

Figure 14. Optical micrograph of the PVC sample 1 dehydrochlorinated (a) up to an extend of $70 \%$ and (b) totally dehydrochlorinated 

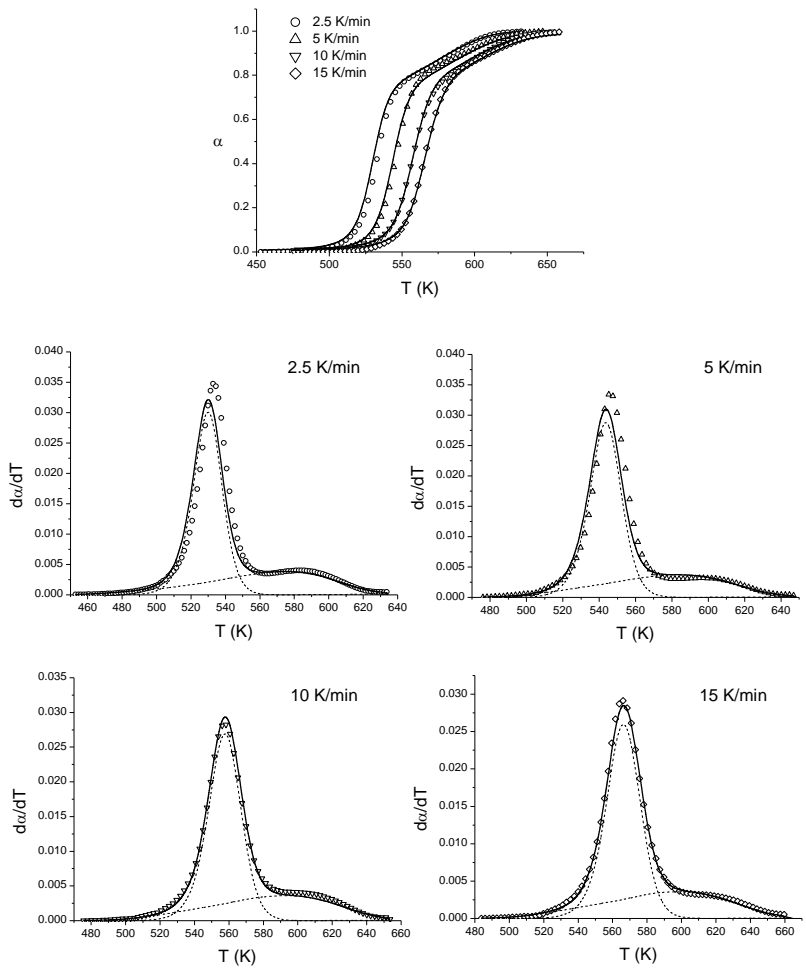

Figure 1. Thermogravimetric curves in its integral (top) and differential (bottom) forms corresponding to the thermal dehydrochlorination of PVC sample 1 obtained under different linear heating rates. The dots are the experimental curves while solid lines are the theoretical ones simulated using the kinetic parameters obtained from the analysis. The contribution of the two processes to the overall reactions is represented by the dashed lines. 


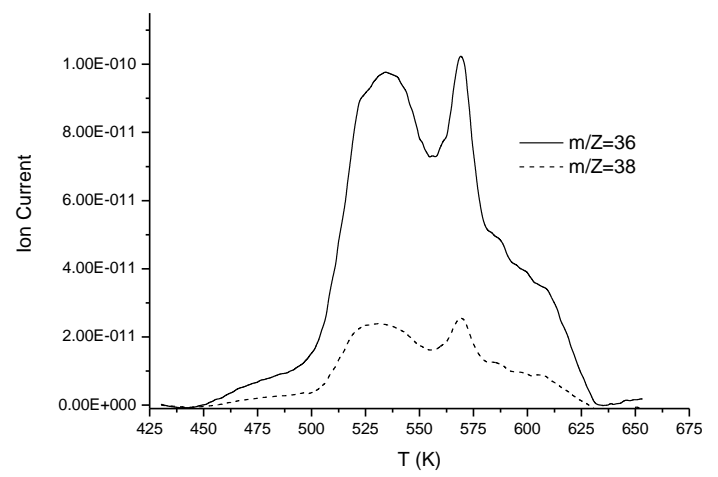

Figure 2. Ion current corresponding to $\mathrm{HCl}$ mass signals recorded by mass spectrometry as a function of the temperature for sample 1 at a heating rate of $10 \mathrm{~K} \mathrm{~min}^{-1}$. 


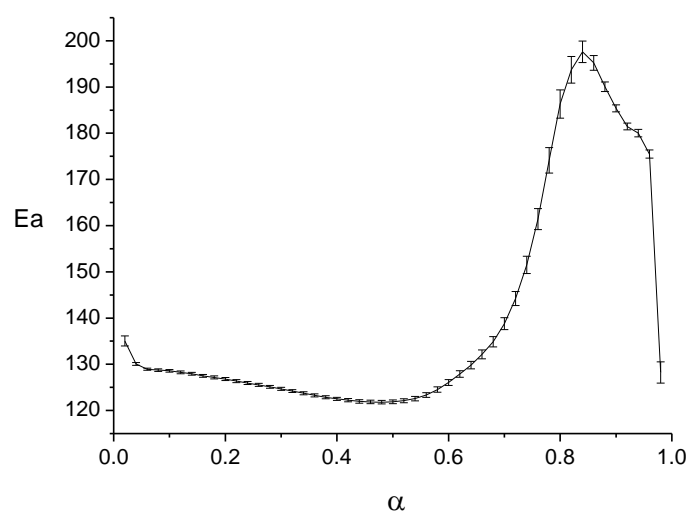

Figure 3. Activation energy values as a function of the reaction progress obtained by means of the model free method for the PVC sample 1. 


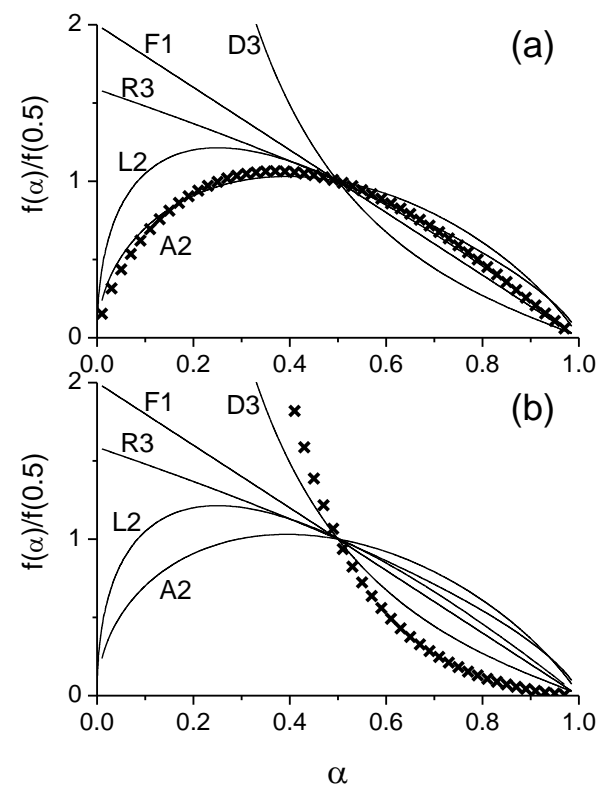

Figure 4. Comparison of the modified Sestak-Berggren $f(\alpha)$ functions resulting from the kinetic analysis (points) with some of the conversion functions often used in the literature and listed in Table 1 (lines). a) first process and b) second process. 

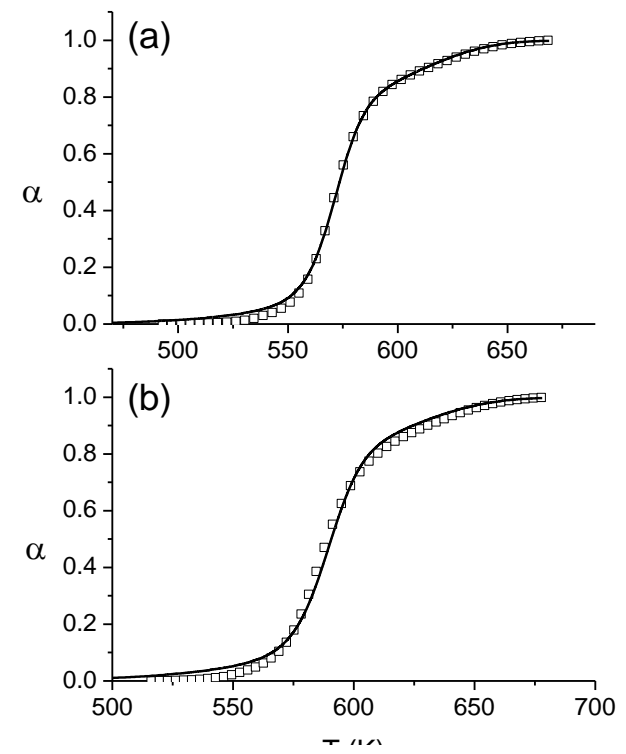

Figure 5. Thermogravimetric curves obtained under linear heating rate conditions at (a) 20 and (b) $40 \mathrm{~K}$ $\min ^{-1}$ for the PVC sample 1. Experimental curves are represented by points while the theoretical reconstructed ones are represented by lines. 


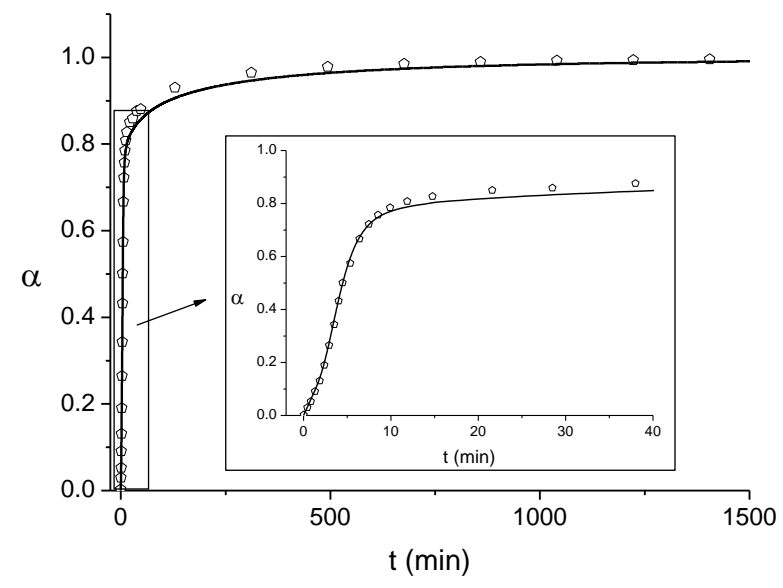

Figure 6. Isothermal experiment at $543 \mathrm{~K}$ for the PVC sample 1. Experimental curve is represented by points while the theoretical reconstructed one is represented by a line. The inset figure shows a detail of the initial part of the degradation curve. 


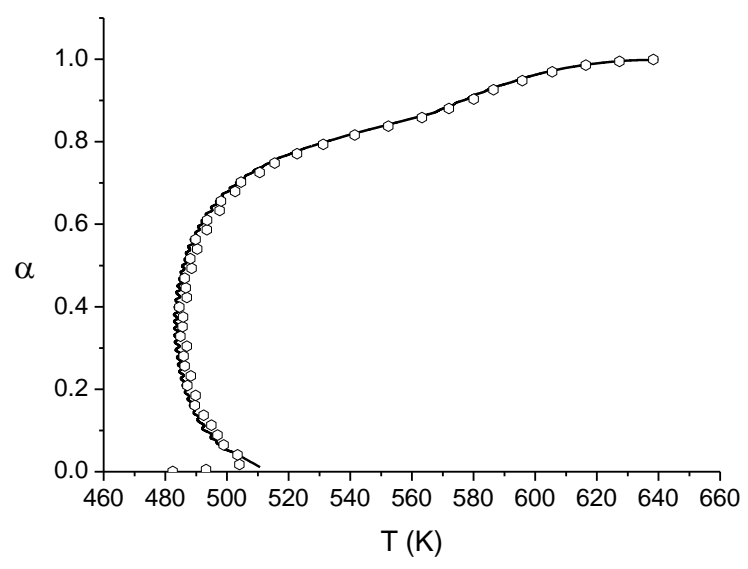

Figure 7. Sample controlled thermal analysis curve resulting from the thermal dehydrochlorination of PVC sample 1 at a constant reaction rate of $610^{-3} \mathrm{~min}^{-1}$. Experimental curve is represented by points while the theoretical reconstructed one is represented by a line. 

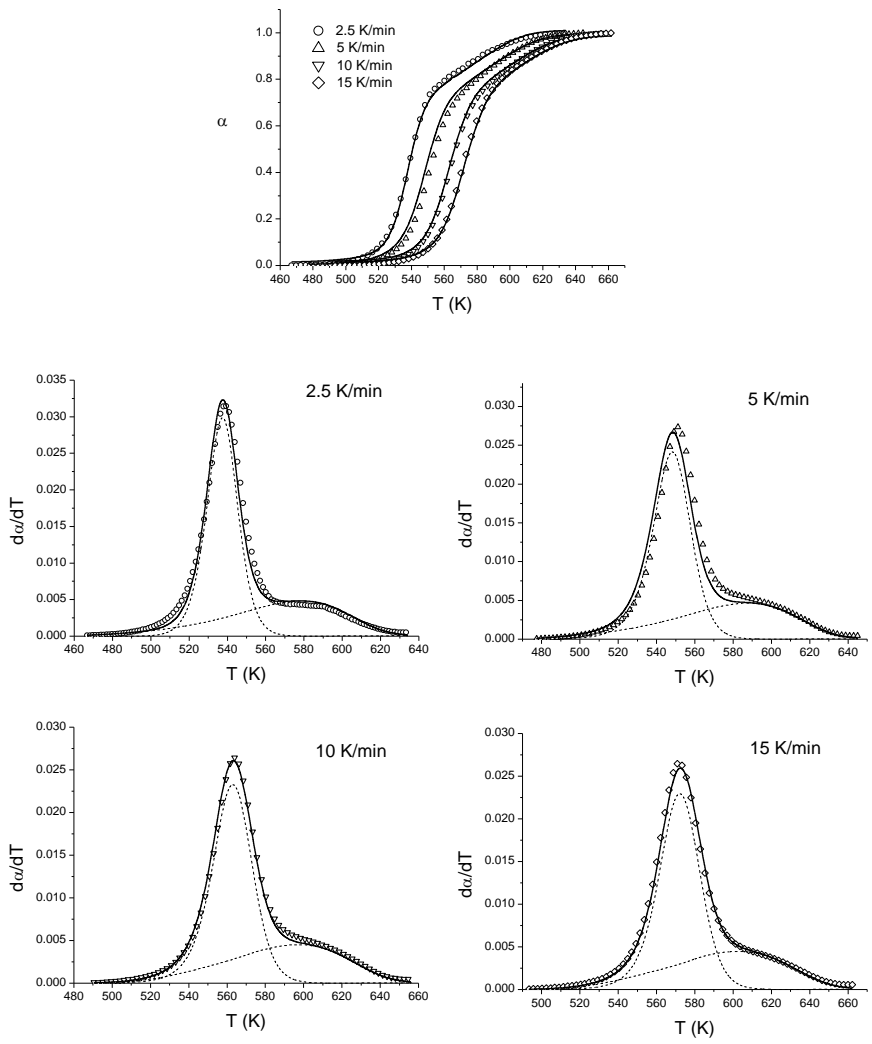

Figure 8. Thermogravimetric curves in its integral (top) and differential (bottom) forms corresponding to the thermal dehydrochlorination of PVC sample 2 obtained under different linear heating rates. The dots are the experimental curves while solid lines are the theoretical ones simulated using the kinetic parameters obtained from the analysis. The contribution of the two processes to the overall reactions is represented by the dashed lines. 


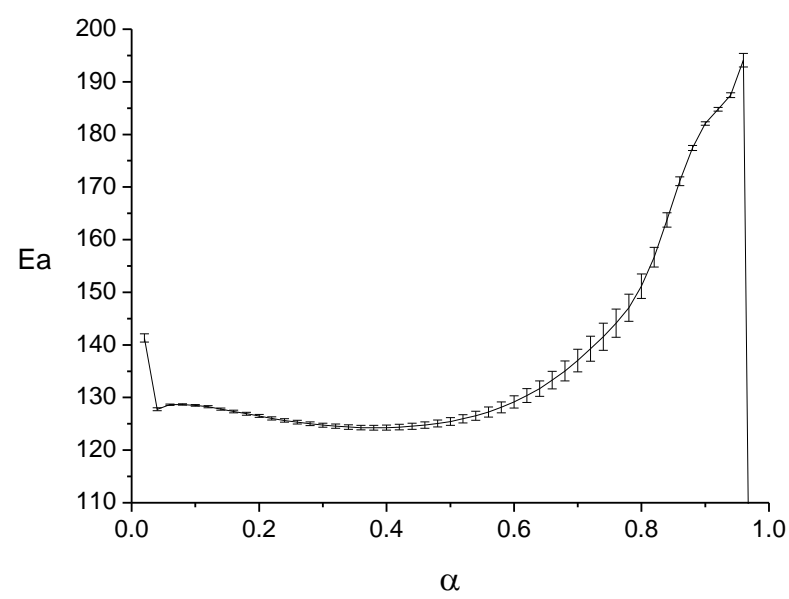

Figure 9. Activation energy values as a function of the reaction progress obtained by means of the model free method for the PVC sample 2. 


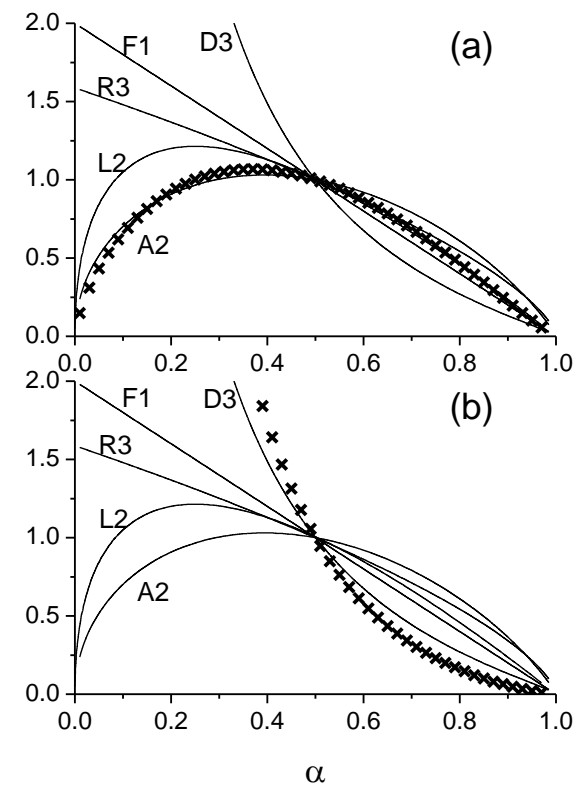

Figure 10. Comparison of the modified Sestak-Berggren $f(\alpha)$ functions resulting from the kinetic analysis (points) with some of the conversion functions often used in the literature and listed in Table 1 (lines). a) first process and b) second process. 

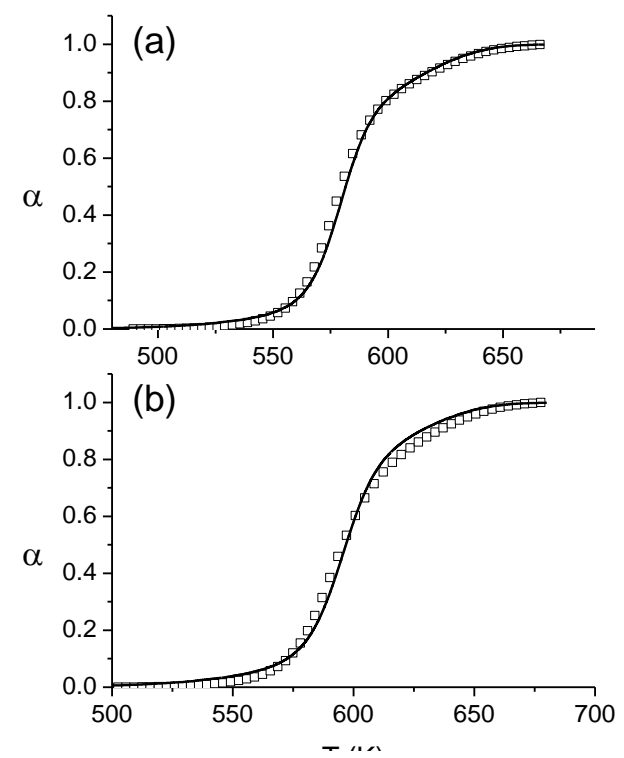

Figure 11. Thermogravimetric curves obtained under linear heating rate conditions at (a) 20 and (b) 40 $\mathrm{K} \min ^{-1}$ for the PVC sample 2. Experimental curves are represented by points while the theoretical reconstructed ones are represented by lines. 


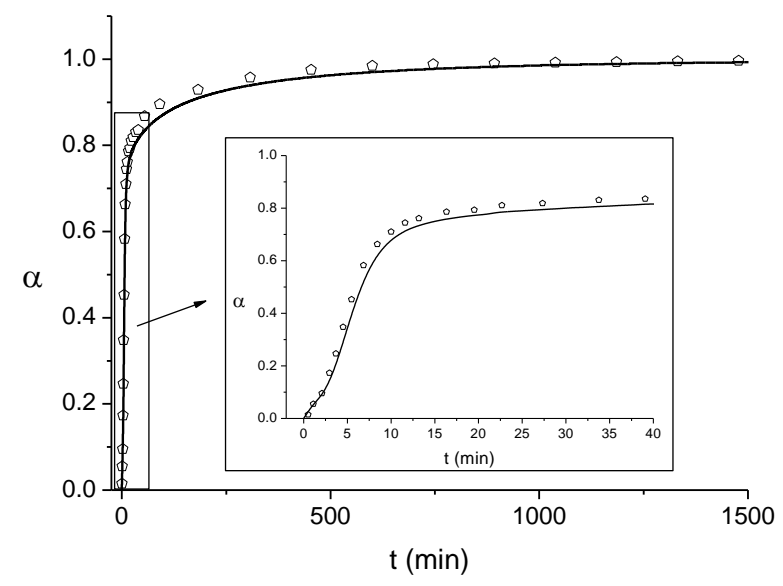

Figure 12. Isothermal experiment at $543 \mathrm{~K}$ for the PVC sample 2. Experimental curve is represented by points while the theoretical reconstructed one is represented by a line. 


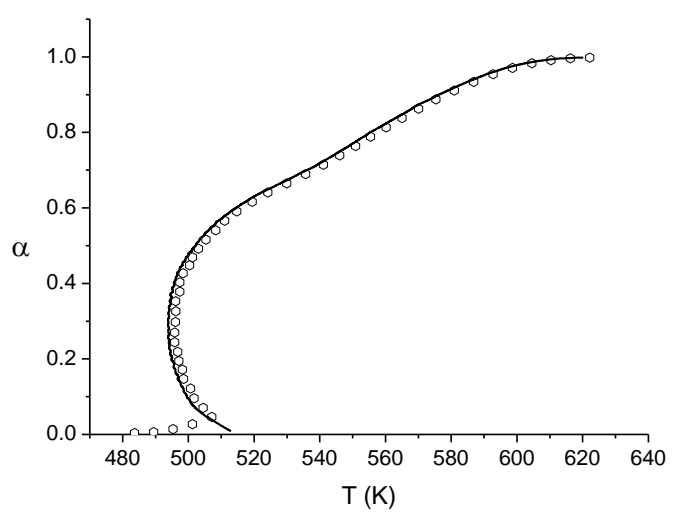

Figure 13. Sample controlled thermal analysis curve resulting from the thermal dehydrochlorination of PVC sample 2 at a constant reaction rate of $610^{-3} \mathrm{~min}^{-1}$. Experimental curve is represented by points while the theoretical reconstructed one is represented by a line. 

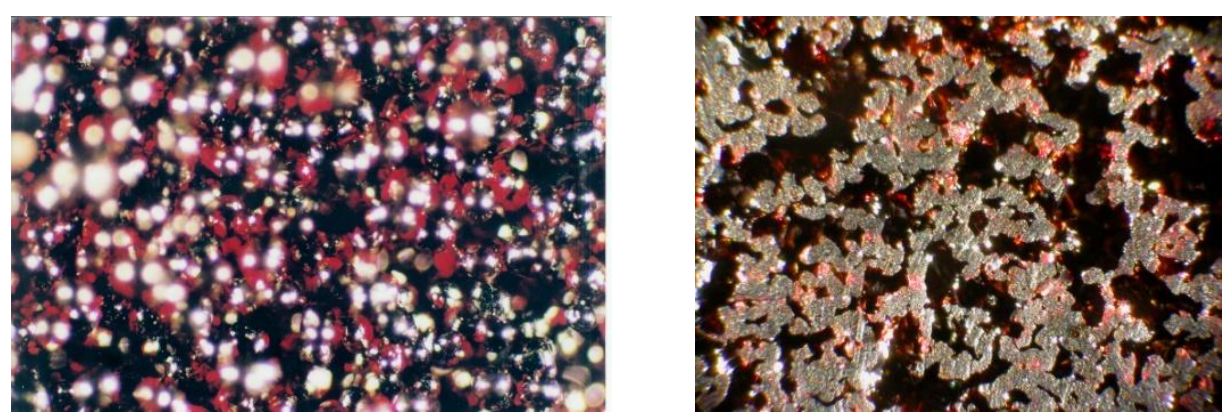

Figure 14. Optical micrograph of the PVC sample 1 dehydrochlorinated (a) up to an extend of $70 \%$ and (b) totally dehydrochlorinated 